EPJ Web of Conferences 19, 09002 (2012)

DOI: $10.1051 /$ epjconf/20121909002

(C) Owned by the authors, published by EDP Sciences, 2012

\title{
Astrometric surveys: Solving the Milky Way puzzle with Gaia
}

\author{
A.G.A. Brown ${ }^{\mathrm{a}}$ \\ Leiden Observatory, Leiden University, PO Box 9513, 2300 RA Leiden, The Netherlands
}

\begin{abstract}
In this contribution I provide a brief summary of ongoing and future astrometric surveys and then focus on presenting the current status and expected scientific performance of the Gaia mission, scheduled for launch in 2013.
\end{abstract}

\section{ASTROMETRIC SURVEYS OF OUR GALAXY}

The results from the Hipparcos mission are a powerful demonstration of the value of high accuracy all-sky astrometry for studies of the structure and formation history of the Milky Way. The results from the Sloan Digital Sky Survey on the other hand show the power of surveys covering large fractions of the volume of our Galaxy with uniform and high quality photometry. We are now poised to enter an era of surveys that will enable us to combine the power of high accuracy astrometry with a large reach within the Milky Way. The resulting map of the Galaxy's phase space and the characterization of its stellar populations will ultimately enable us to assemble the puzzle of the Milky Way.

Past, present, and future astrometric surveys were reviewed recently for the IAU Symposium 'A Giant Step: from Milli- to Micro-arcsecond Astrometry' [1]. An overview of the various programmes was given as well as a description of parameter space that defines astrometric surveys. The latter was used to try and identify gaps in the present astrometric programmes and thus fruitful directions for future astrometric surveys. Figure 1 is an update of figure 2 in [1] and shows that with the cancellation of the SIM Lite mission the only astrometric survey in the optical that covers the $10 \mu$ as to 1 mas accuracy range will be Gaia. Thus as illustrated in section 3 the Gaia mission will play a central role in assembling the puzzle of our galaxy. The final Gaia catalogue is not expected until $\sim 2021$, with a first data release foreseen in 2015-2016. Nevertheless there is no reason to sit and wait for the Gaia catalogue. The many contributions to this conference show that there is plenty of high-quality survey data to exploit now, before the Gaia catalogue arrives. Examples of surveys that already allow large scale studies of the Galaxy are the Sloan digital sky survey (with good proper motions derived for 'strip 82'), the Hipparcos catalogue and its re-reduction, the Geneva-Copenhagen Survey, the Tycho-2 catalogue, the RAVE survey, and soon the first Pan-Starrs survey results. In addition to these wide area surveys there are targeted surveys providing very high accuracy astrometry, such as the surveys of the Galactic centre described by Schödel in this volume, and the Bessel survey [2] providing $10 \mu$ as level parallaxes for individual masers in the Galaxy, allowing accurate studies of the Galactic disk structure and kinematics.

In the rest of this contribution I will focus on the Gaia mission. I will summarize the current status of the mission and the most recent performance predictions, as well as highlight some of the less obvious ways in which Gaia will impact studies of the Milky Way.

\footnotetext{
ae-mail: brown@strw.leidenuniv.nl
}

This is an Open Access article distributed under the terms of the Creative Commons Attribution-Noncommercial License 3.0, which permits unrestricted use, distribution, and reproduction in any noncommercial medium, provided the original work is properly cited. 


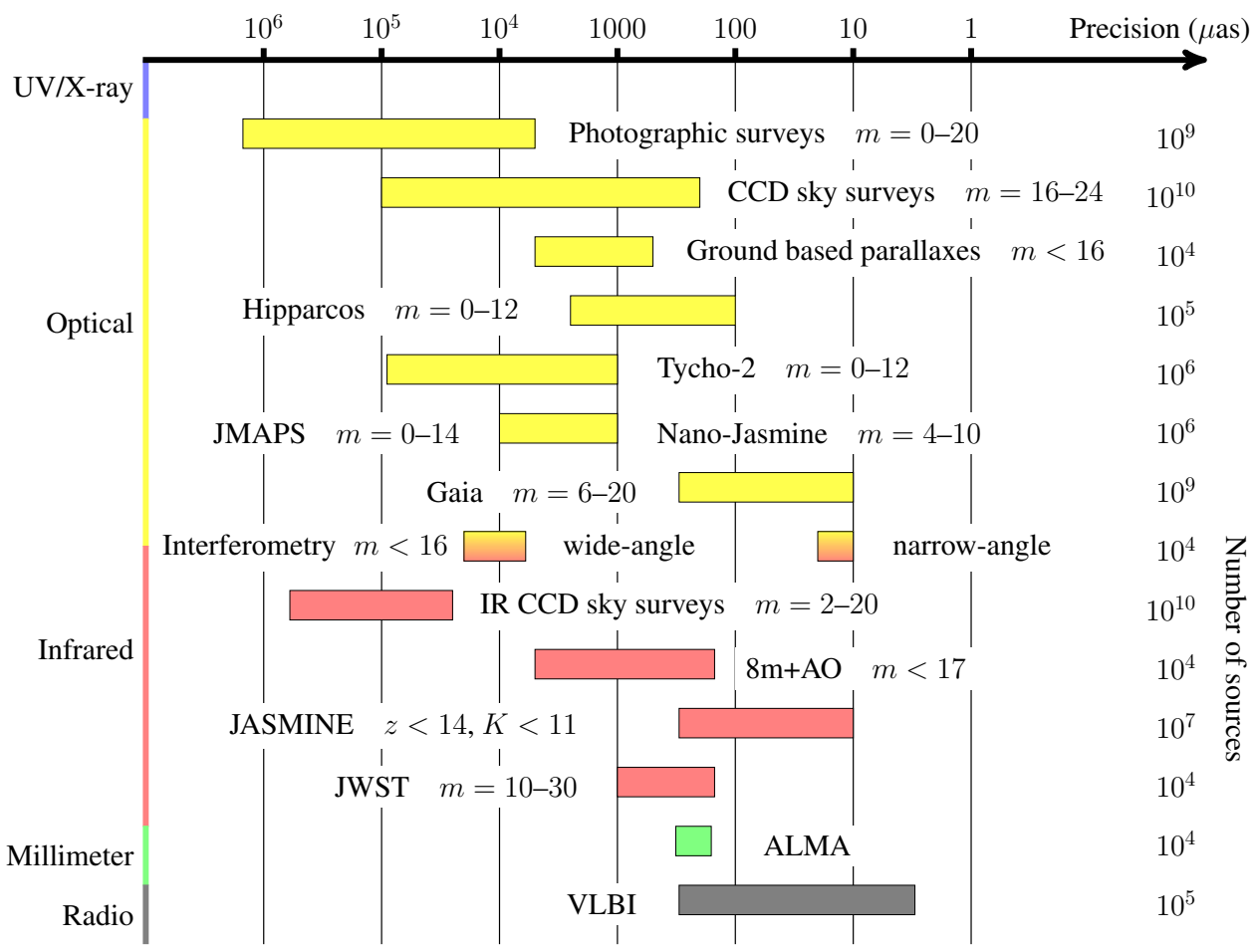

Figure 1. Graphical overview of astrometric surveys. The surveys are characterized by a number of parameters: wavelength range, depth, magnitude range, precision reached, number of objects surveyed. The precision refers to all astrometric parameters (position, proper motion, parallax), where typically the proper motion precisions are numerically smaller than parallax precision. Implicit in the proper motion precision is also the time baseline of a given astrometric survey. The shading of the boxes and the right vertical axis serves to aid the eye in segregating the wavelength domains. The references for the survey parameters can be found in [1].

\section{GAIA MISSION STATUS AND PERFORMANCE PREDICTIONS}

Gaia is the European Space Agency mission that will carry out an all-sky astrometric, photometric, and spectroscopic survey - observing every object brighter than $20^{\text {th }}$ magnitude - amounting to about 1 billion stars, galaxies, quasars and solar system objects. At the time of writing this contribution the Gaia spacecraft and payload are in an advanced stage of assembly with extensive tests of the integrated scientific payload expected to take place by mid-2012. Gaia is on track for a launch by mid-2013.

The scientific power of Gaia rests on the combination of three desirable qualities in a single mission: (1) the ability to make very accurate (global and absolute) astrometric measurements; (2) the capability to survey large and complete (magnitude limited) samples of objects; and (3) the matching collection of synoptic and multi-epoch spectrophotometric and radial velocity measurements (cf. [3]). The range of science questions that can be addressed with such a data set is immense and Gaia will revolutionize studies of the Milky Way. Much of the Gaia science case can be found in this volume and the proceedings of the conferences 'The Three-Dimensional Universe With Gaia' [4] and 'Gaia: At the Frontiers of Astrometry' [5].

The astrometric measurements are collected employing a wide photometric band (the Gaia $G$ band) which covers the range 330-1000 nm. Multi-colour photometry will be obtained for all objects by means of low-resolution spectrophotometry. The photometric instrument consists of two prisms dispersing all the light entering the field of view. One disperser - called BP for Blue Photometer - operates in the 


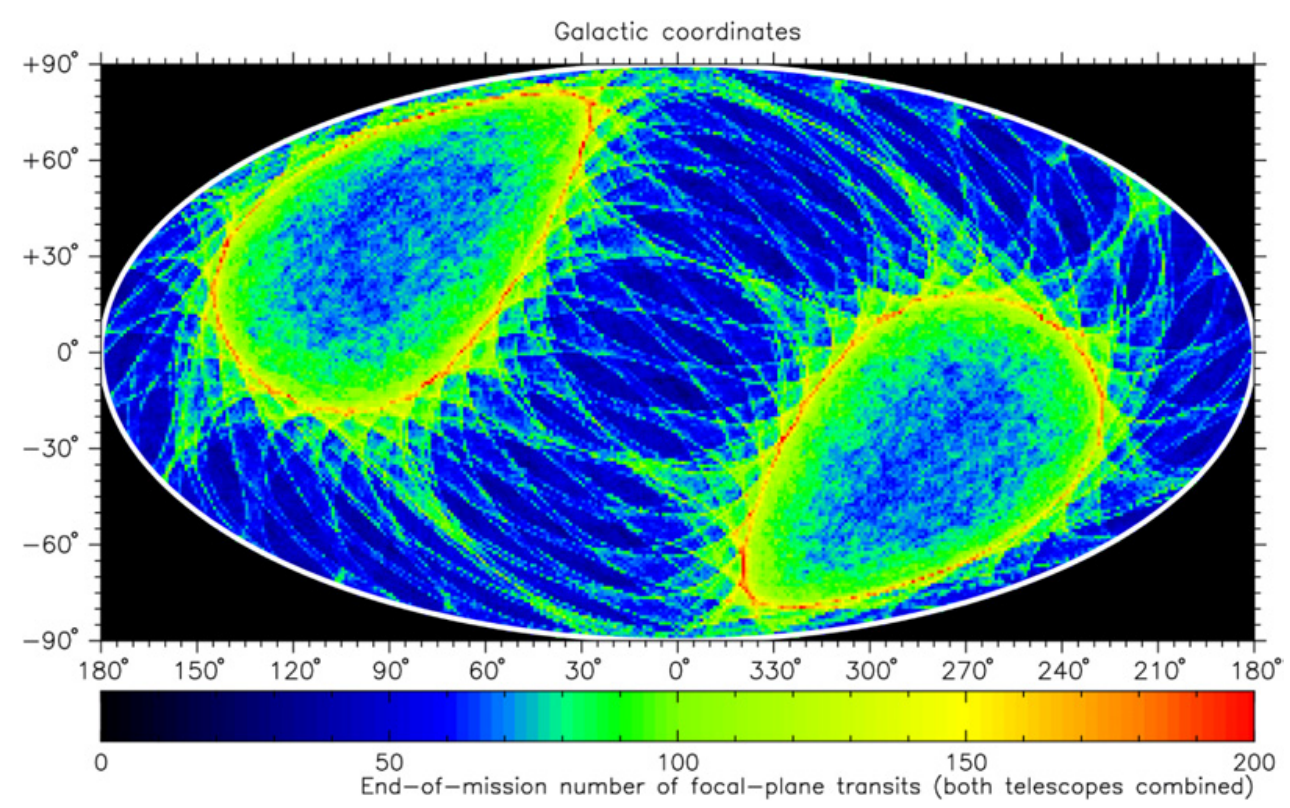

Figure 2. The predicted sky coverage of Gaia in Galactic coordinates. The colours indicate the number of times a particular region on the sky is observed. By using the Galactic coordinate grid one can appreciate which regions of the Milky Way will be observed more often. The two annuli around the ecliptic poles where most of the observations are reflect the constant $45^{\circ}$ angle between the Gaia spin axis and the direction from the spacecraft to the sun. Image courtesy J. de Bruijne and ESA.

wavelength range 330-680 nm; the other - called RP for Red Photometer - covers the wavelength range $640-1000 \mathrm{~nm}$. In addition radial velocities with a precision of $1-15 \mathrm{~km} \mathrm{~s}^{-1}$ will be measured for all objects to $17^{\text {th }}$ magnitude, thus complementing the astrometry to provide full six-dimensional phase space information for the brighter sources. The radial velocity instrument (RVS) is a near-infrared (847$874 \mathrm{~nm}, \lambda / \Delta \lambda \sim 11000$ ) integral-field spectrograph dispersing all the light entering the field of view.

The focal plane of Gaia comprises an array of 106 CCD detectors which serve the three instruments mentioned above. The observing programme is based on the autonomous on-board detection of celestial sources, which is unbiased and complete to $G=20(V \sim 20-22)$. Gaia will be located at L2 and scan the sky with its two telescopes by continuously spinning around the axis perpendicular to the two lines of sight. The spin axis in addition makes a precessing motion around the spacecraft-sun direction, and as a result Gaia will scan the whole sky roughly every 6 months. Each celestial source will on average be observed about 80 times during the 5 year mission lifetime with a quasi-regular time sampling. The number of times a source is observed is not uniform across the sky as illustrated in figure 2 . The regions in the annuli located $45^{\circ}$ away from the ecliptic poles are observed most often, while the regions around the ecliptic are covered less often. The number of stars in the Gaia catalogue is estimated to be $\sim 7 \times 10^{5}$ to $G=10,48 \times 10^{6}$ to $G=15$ and $1.1 \times 10^{9}$ to $G=20$. About 60 million stars are expected to be seen as binary or multiple systems by Gaia, among which about $10^{6}-10^{7}$ eclipsing binaries. In addition the catalogue will contain astrometry and photometry for $\sim 3 \times 10^{5}$ solar systems bodies, $\sim 5 \times 10^{5}$ quasars, and some $10^{6}-10^{7}$ galaxies. The sky survey by Gaia will also produce the most accurate optical all-sky map ever, with an angular resolution comparable to that of the Hubble Space Telescope.

The astrometric performance of Gaia is summarized in figure 3, which shows the sky averaged parallax standard errors as a function of $V$-band magnitude. This figure illustrates a number of important issues to keep in mind. At the bright end calibration errors will dominate and the parallax accuracies will range from $\sim 5$ to $\sim 14 \mu$ as. At the faint end the behaviour of the errors as a function of $V$ is 


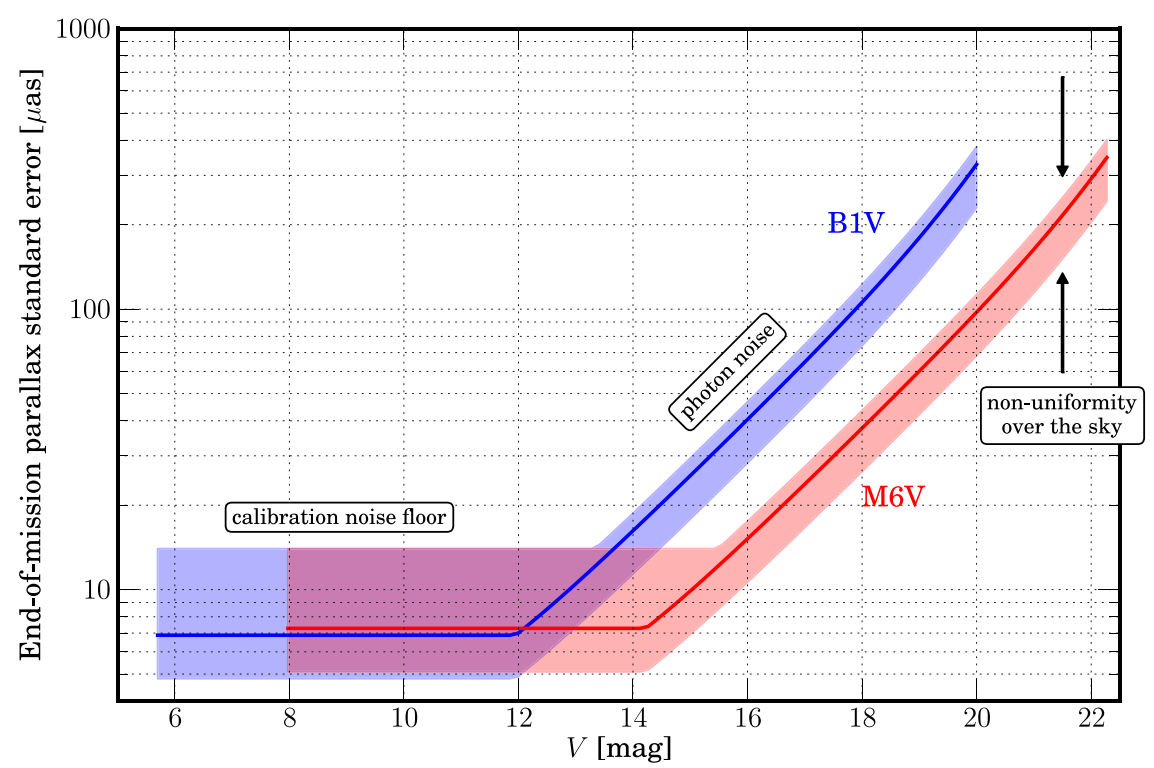

Figure 3. Sky averaged parallax standard errors as a function of magnitude in the $V$-band. The errors are shown for stellar spectral types B1V and M6V. See the text for further explanations of this plot. The figure was made using the performance predictions available through the ESA Gaia web pages.

dictated by the photon noise. The parallax accuracies are shown for an early and a late spectral type to illustrate that at a given $V$ the astrometric accuracies are better for red stars. As a function of $G$ the differences are negligible. The bands around the average relations reflect the uncertainty on calibration errors at the bright end and the variation in sky coverage at the faint end. Note furthermore that expressed in $V$ the survey limits for red stars are different, being fainter at both ends. For M6V stars the limits are $8 \lesssim V \lesssim 22$. The parallax accuracy predictions include a rough estimate of the effects of radiation damage and a $20 \%$ margin (factor 1.2) to account for unmodelled effects. The standard errors in position and proper motion can be obtained by applying factors of $\sim 0.5$ and $\sim 0.7$, respectively, to the parallax standard errors.

The velocities of the stars perpendicular to the line of sight can be inferred from the proper motion and parallax. The expected accuracies on the transverse motion are around $1 \mathrm{~km} \mathrm{~s}^{-1}$ at a distance of $1 \mathrm{kpc}$, and range from $\sim 1-20 \mathrm{~km} \mathrm{~s}^{-1}$ at $3 \mathrm{kpc}$. At $10 \mathrm{kpc}$ the transverse motions for $\mathrm{K}$ giants are expected to be around 20 to $30 \mathrm{~km} \mathrm{~s}^{-1}$. To complement the transverse motions Gaia will collect radial velocities for the brightest 150 million stars in the survey. The predicted accuracies range from 1 to $20 \mathrm{~km} \mathrm{~s}^{-1}$ with a rather strong dependence on spectral type. For FGK stars accuracies better than $5 \mathrm{~km} \mathrm{~s}^{-1}$ can be achieved to $V \sim 16$, while for earlier spectral types this limit is already reached at $V \sim 12$.

A stereoscopic and kinematic map of the Milky Way is only useful when complemented by a detailed characterization of the age distributions and chemical compositions of the stellar populations. To this end the multi-colour photometry and RVS spectra for the brighter stars can be used. Figure 4 shows simulated Gaia spectrophotometric observations of main sequence stars. The different spectral types are clearly distinguishable and from these observations the astrophysical parameters of the stars can be derived, including $T_{\text {eff }}, A_{V}, \log g,[\mathrm{M} / \mathrm{H}]$, and $[\alpha / \mathrm{Fe}]$ for the brighter stars. In addition the detailed analysis of the Ca triplet region from the RVS spectra is expected to deliver the following diagnostics: rotational velocities, atmospheric parameters, interstellar reddening $(V<13,5$ million stars), and abundances ( $V<12,2$ million stars). In addition the RVS spectra can be used to study 

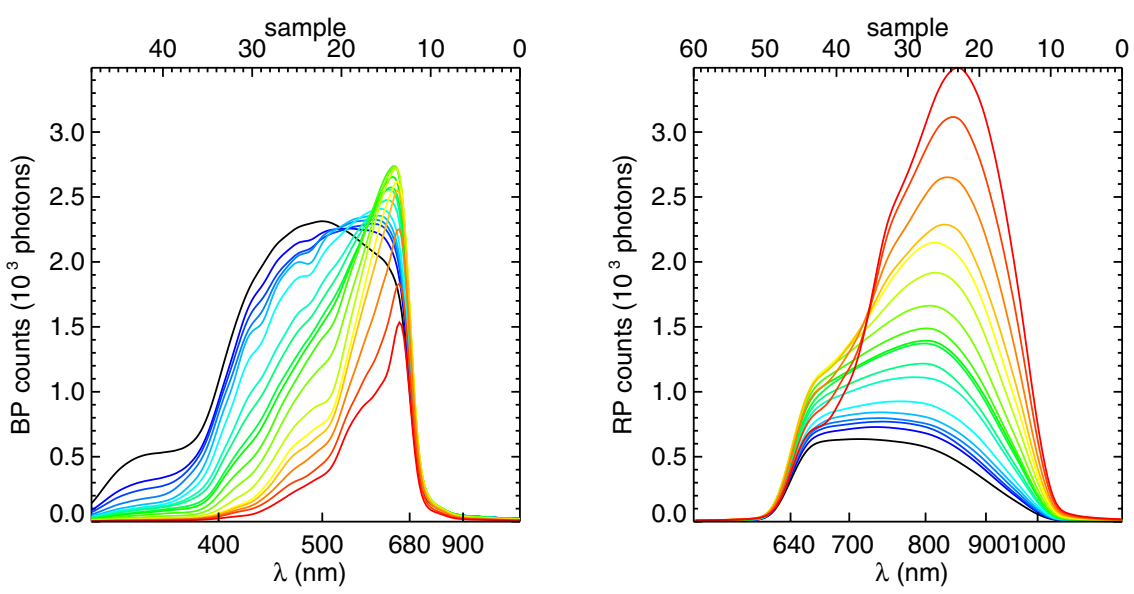

Figure 4. The response of Gaia's blue and red photometers (left and right panel respectively) to main sequence stars of spectral types varying from $\mathrm{O} 5 \mathrm{~V}$ (blue lines) to M5V (red lines). Input stellar spectral energy distributions were provided by V. Straižys.

binary stars, where about $10^{6}$ spectroscopic and $10^{5}$ eclipsing binaries are expected, a quarter of which will be double-lined spectroscopic binaries, allowing mass estimates.

Much more information on the expected performance and catalogue contents of Gaia than can be provided here can be found on the ESA Gaia web pages (http://www.rssd.esa.int), in particular in the 'Science Performance' section. The latter provides, among others, background information on the instruments and the error modelling, interpolation tables and formulae for simulating the errors and the predicted variations thereof over the sky, transformations from the Johnson and Sloan systems to Gaia photometric system, and references to the relevant literature. The information on these pages allows one to precisely simulate the performance of Gaia in order to prepare for the scientific exploitation of the data. These web pages will be updated with better estimates of the expected accuracies on derived products such as stellar astrophysical parameters. Over the next few years the Gaia Data Processing and Analysis Consortium together with the Gaia Science Team will also make available simulated Gaia catalogues containing the billion expected sources so that the astronomical community can prepare for the scientific exploitation of the Gaia catalogue.

\section{GAIA'S IMPACT ON SOLVING THE PUZZLE OF THE MILKY WAY}

The scientific impact of the Gaia data on our understanding of the structure and formation history of the Milky Way will be unprecedented with hopefully many interesting surprises awaiting us. I will not attempt to summarize the expected science harvest here, but instead point out a number of the less obvious ways in which Gaia will impact Galactic astronomy.

First of all it is important to realize that out to a distance of about $2.5 \mathrm{kpc}$ from the sun parallaxes can be determined to better than 1 per cent, covering about 11 million stars. This will enable an extremely accurate calibration of luminosities and distance indicators across the Hertzsprung-Russell diagram. This information can then be used to extend the reach of Gaia by employing well-calibrated photometric distances instead of parallaxes at the faint end of the survey. Secondly, keep in mind that Gaia is a multiepoch survey of the sky and is expected to observe about 20 million classical variables, including 1-5 million eclipsing binaries, about 5000 Cepheids and 70000 RR Lyra variables. The latter will enable us to probe Galactic structure out to $\sim 75 \mathrm{kpc}$. 
In terms of dissecting the structure of the Milky Way and understanding the fossil record of its formation Gaia has a number of unique capabilities not provided by any other large sky survey. Gaia will directly map Galactic phase space (3D distances and motions) over the full sky and a large volume. In addition ages and compositions of stellar populations can be mapped, an endeavour that will be very much enhanced by the large ground-based spectroscopic surveys described by Steinmetz in this volume. Gaia data allow a mapping of dynamical quantities such as energy and angular momentum, which is required to disentangle the formation history of the inner halo where dynamical mixing precludes the detection of substructure as features in sky maps. Gaia will also enable us to study other 'solar neighbourhoods', as described by Antoja at this conference, something that can not (easily) be done with presently available data. The combination of Gaia parallaxes, interstellar reddening measures and data on the Milky Way's ISM will enable us to map the ISM accurately in three dimensions. This will add very powerful constraints on any model we construct of the Milky Way.

Although the vast majority of stars observed will be within about $10 \mathrm{kpc}$ from the sun, one should keep in mind that Gaia will observe every source to $G=20$. This means that individual bright giants will be observed even in M31 and in particular that a significant fraction of the population of nearby dwarf galaxies (to $V \sim 20-22$ depending on the colour of the star) will be probed by Gaia. Accurate distances and motions of these galaxies can thus be determined.

In relation to large ground based surveys of the sky Gaia will also have a tremendous impact. It will enable excellent calibrations of standard candles and photometric distance indicators which in the ground based surveys can be seen to distances 10 times further than can be reached by Gaia. In addition Gaia will provide an extremely accurate reference frame against which astrometry can be done from the ground. For example in the LSST science book it is illustrated how the proper motion and parallax errors form a continuation from the Gaia errors to fainter magnitudes. This means that we will have access to Hipparcos quality astrometry out to $24^{\text {th }}$ magnitude. Finally, Gaia parallaxes, even when of relatively poor quality, will provide the possibility to perform dwarf/giant separation which is important in target selection for spectroscopic surveys.

\section{PLANS FOR THE PUBLICATION OF GAIA DATA}

The astronomical community is eagerly looking forward to the Gaia catalogue which is expected to be ready in its final form around 2021, 8 years after launch. This is however ten years from today so in keeping with modern practice, the Gaia community is developing plans for early and multiple data releases. Each data release is planned to be made public world-wide immediately and the release schedule is currently under discussion. The data releases will be increasingly complex in scope, starting from an all-sky catalogue of accurate positions, including magnitudes and colours for each source. Subsequently data such as parallaxes, proper motions, radial velocities, and multicolour photometry will be added. Lastly, derived quantities are added such as stellar astrophysical parameters (including ages), variable star classification, binary and multiple system parameters (orbital solutions, masses). Detailed error information will be provided, including parameter covariances and estimates of source to source correlations.

Scientifically exploiting the huge and complex catalogue that will be produced with Gaia will not be trivial. Hence the Gaia community is now in the process of defining the way that Gaia data will be made accessible to astronomers. This includes developing the data archiving and querying systems, as well as producing detailed documentation and providing sophisticated data analysis tools, such as visualization software for high dimensional data. In addition the aim is to make the data available in a form that allows reprocessing of and adding to (parts of) the Gaia data in order to maximize the science return. Examples are advanced searches for substructure in the Galactic halo by jointly considering the intermediate data on groups of stars, or incorporating future information such as complementary 
Assembling the Puzzle of the Milky Way

ground based spectroscopy. This is not a closed process taking place within the Gaia community. Ideas on the Gaia catalogue are very welcome and are in fact currently being solicited from the astronomical community through a requirements gathering process.

\section{References}

[1] Brown A.G.A. in Proceedings of IAU Symposium 248: A Giant Step: from Milli- to Microarcsecond Astrometry (Cambridge University Press, Cambridge 2008) pp. 567-576

[2] Brunthaler A., et al., Astronomische Nachrichten 332, (2011) 461-466

[3] Lindegren L., in Proceedings of IAU Symposium 248: A Giant Step: from Milli- to Micro-arcsecond Astrometry (Cambridge University Press, Cambridge 2008) pp. 217-223

[4] Turon C. O'Flaherty K.S., Perryman, M.A.C., The Three-Dimensional Universe with Gaia (ESA Special Publication 576, 2005)

[5] Turon C., Meynadier F., Arenou F., Gaia: At the Frontiers of Astrometry (EAS Publications Series Vol. 45, EDP Sciences 2011) 